\title{
Sinopsis de la familia Amaryllidaceae en Colombia
}

\section{Synopsis of the family Amaryllidaceae in Colombia}

\author{
Fernando Alzate, Manuela Lesmes, Natalie Cortés, Santiago Varela y Edison Osorio
}

\section{Resumen}

La familia Amaryllidaceae ha sido señalada en diferentes análisis como un grupo monofilético, en el cual se reconocen tres grandes clados, tratados como subfamilias (Agapanthoideae, Allioideae y Amaryllidoideae). Revisamos especímenes de la familia y exploramos diferentes áreas del país donde se tuvieran registros previos o potencialidad de ocurrencia del grupo, para determinar la diversidad de esta familia en Colombia. Amaryllidaceae está representada en Colombia por 18 géneros y 48 especies, de los cuales 11 géneros y 27 especies son nativos, siendo 9 especies endémicas de Colombia. Las áreas donde se encuentra la mayor diversidad de especies son la cordillera Occidental y Oriental y el Chocó biogeográfico, especialmente en los departamentos de Cauca, Cundinamarca y Valle del Cauca. Presentamos una definición actualizada de la familia, considerando la circunscripción vigente, y describimos los géneros presentes en Colombia, así como el inventario de las especies y su distribución.

Palabras clave. Andes. Asparagales. Horticultura. Plantas neotropicales.

\begin{abstract}
The family Amaryllidaceae has been postulated as a monophyletic group in different analyses, in which three big clades are recognized as subfamilies (Agapanthoideae, Allioideae and Amaryllidoideae). We examined specimens of the family and explored different areas of the country where there were previous records or potential occurrence of the group, in order to determine the diversity of this family in Colombia. The Amaryllidaceae are represented in Colombia by 18 genera and 48 species, of which 11 genera and 27 species are native, including 9 species endemic to the country. The areas with the largest species diversity are the Western and Eastern Cordillera, and the biogeographic Chocó, especially in the departments of Cauca, Cundinamarca and Valle del Cauca. We present an updated definition of the family, considering its current circumscription, and describe the genera occurring in Colombia, as well as the inventory of the species and their distribution.
\end{abstract}

Keywords. Andes. Asparagales. Horticulture. Neotropical plants. 


\section{Introducción}

La circunscripción del orden Asparagales ha sido objeto de cambios en cuanto a los clados que lo componen, lo cual se advierte en las clasificaciones planteadas en APG I (1998), donde se reconocen 29 familias para el orden, y APG IV (2016), en la que este número se reduce a 14. La familia Amaryllidaceae ha sido uno de los taxones del orden que ha sufrido mayores modificaciones en cuanto a su delimitación taxonómica y a los taxones intrafamiliares que incluye.

Amaryllidaceae es un grupo monofilético, reconocido como familia desde inicios del siglo XIX, y en la delimitación actual incluye cerca de 1650 especies en 73 géneros. Chase et al. (2009) reconocen tres grandes clados para Amaryllidaceae, representados en las subfamilias Agapanthoideae, Allioideae y Amaryllidoideae, esta última la más rica en especies en Colombia.

Las Amaryllidaceae se distribuyen principalmente en zonas tropicales y holárticas, con una considerable diversidad en África y Suramérica (Meerow \& Snijman, 1998), seguida de Asia y Australia. La diversidad de este grupo de plantas en Colombia no está bien establecida; además, hay un considerable número de especies introducidas con variantes morfológicas que generan incertidumbre en cuanto a su identidad taxonómica, lo que ha resultado en una menor certeza en cuanto al número de especies que se encuentran en el país. En el Catálogo de las plantas vasculares de Colombia (Bernal et al., 2015) se reportan 16 géneros y 42 especies de Amaryllidaceae.

Con la finalidad de establecer la diversidad de especies que tiene esta familia de plantas en Colombia, se desarrolló un amplio muestreo en diferentes zonas del país, complementado con la revisión de numerosas colecciones de los principales herbarios colombianos.

\section{Materiales y métodos}

La revisión taxonómica de la familia Amaryllidaceae se realizó con base en colecciones de herbario y en especímenes recolectados durante trabajos de campo realizados en áreas donde, de acuerdo con la información recopilada de los especímenes de herbario, se tuviera una considerable diversidad para la familia en Colombia (sur de la cordillera Occidental en las cercanías del Macizo colombiano, parte norte de la cordillera Occidental, centro de la cordillera Central, Chocó biogeográfico, altiplano cundiboyacense, Santander y Orinoquia). Se revisaron cerca de 1200 especímenes en los principales herbarios nacionales, los cuales fueron: COAH, COL, CUVC, FAUC, HUA, HUQ, JAUM, MEDEL, PSO y VALLE. También fueron consultadas las colecciones de seis herbarios extranjeros: F, MO, NY, K y US (abreviaturas de acuerdo con Thiers, 2018). Los especímenes tipo disponibles fueron revisados, tanto en los herbarios consultados, como en las bases de datos disponibles, como JSTOR Global Plants.

Las observaciones sobre caracteres morfológicos se hicieron mediante un estereoscopio binocular Nikon (Nikon, Tokio), y para las descripciones se siguió la terminología de Font Quer (2001) y Beentje (2016). Los nombres comunes fueron tomados de las etiquetas de las colecciones de herbario y del diccionario de nombres comunes de las plantas de Colombia (Bernal et al., 2017).

\section{Resultados}

La revisión de especímenes y las exploraciones de campo llevadas a cabo permitieron establecer la ocurrencia de 18 géneros y 48 especies. 


\section{Amaryllidaceae}

Hierbas perennes, a veces caducifolias durante la antesis (Caliphruria, Phaedranassa, Scadoxus, Allium y algunas especies de Hymenocallis). Bulbos tunicados, ocasionalmente rizomas (Clivia, Tulbaghia, Scadoxus y Agapanthus), geófitos, en algunos casos acuáticos (Crinum), o raíces filamentosas (Pamianthe), perennes o fugaces y fibrosas, creciendo de manera adventicia en la base del bulbo o del tallo. Hojas simples, alternas, dísticas o en roseta espiralada, láminas pecioladas ( $x$ Calicharis, Caliphruria, Eucharis, algunas especies de Hymenocallis, Narcissus, Phaedranassa, Plagiolirion, Scadoxus) o a veces sésiles (Agapanthus, Allium, Nothoscordum,Tulbaghia, Clivia, Crinum, Habranthus, Hippeastrum, Hymenocallis y Zephyranthes), lineares, elípticas, semicirculares, ovadas, lanceoladas, con forma de correa o cóncavas en la subfamilia Allioidae, ocasionalmente con lígula en la base del pecíolo lineal y plano, en ocasiones envolviendo el pseudotallo. Inflorescencias umbeladas o pseudo-umbelas (Pamianthe), escapifloras, o flores solitarias (Zephyranthes, $\mathrm{Ha}$ branthus y algunas especies de Crinum y Narcissus); escapos cilíndricos, fistulosos o sólidos, teretes o angulosos, en algunos casos con dos o más brácteas en la inflorescencia que se traslapan alternamente por los márgenes. Flores 1-500, actinomorfas o raramente zigomorfas (Hippeastrum, Narcissus, Pamianthe, Plagiolirion y Scadoxus), bisexuales; pedicelo no articulado; perigonio con seis tépalos dispuestos en dos verticilos, usualmente connados en la base, formando un tubo infundibuliforme o crateriforme, y ocasionalmente presentando paraperigonio no estaminal ( $\mathrm{Co}-$ rona interna formada por el perigonio sin la participación de los estambres); tépalos de una amplia gama de colores, azules en Agapanthus y blancos en Eucharis; androceo conformado por seis estambres dispuestos en dos o tres verticilos, libres o formando paraperigonio estaminal (copa formada por la fusión parcial o total de los filamentos, dando la apariencia de un verticilo perigonial), los filamentos insertos en la garganta del tubo floral o exsertos (Hymenocallis, Phaedranassa y Scadoxus), separados o connados, ocasionalmente apendiculados, las anteras dehiscentes por hendiduras laterales; gineceo sincárpico; ovario ínfero en Amaryllidoideae y súpero en Agapanthoideae y Allioideae, tricarpelar, trilocular; estilo cilíndrico, estigma trilobado o entero, óvulos de placentación axilar, con nectarios septados. Frutos usualmente cápsulas loculicidas o raramente bayas. Semillas numerosas, carnosas, globosas, aplanadas, aladas, esféricas, angulosas, elipsoides u ovadas, rojas, anaranjadas, azules, marrones o negras (Figura 1).

La familia está representada en Colombia por 18 géneros y 48 especies.

\section{Clave para los géneros de Amaryllidaceae presentes en Colombia}

1. Hojas pecioladas...................................... 2

- Hojas sésiles............................................... 8

2. Estambres libres..........................................3

- Estambres fusionados en paraperigonio estaminal

(copa) .......................................................... 5

3. Fruto en baya...............................Scadoxus

- Fruto en cápsula............................................... 4

4. Paraperigonio no estaminal (corona) presente, tépalos patentes, blancos o amarillos, estambres insertos

Narcissus

- Paraperigonio no estaminal (corona) ausente, tépalos dispuestos en forma tubular, rosados-rojos y amarillos o verdes en la porción distal, estambres marcadamente exsertos Phaedranassa

5. Flores zigomorfas, carentes de aroma......Plagiolirion - Flores actinomorfas, aromáticas. 6

6. Pedicelo 4.5-5 mm de largo............... $x$ Calicharis - Pedicelo 10-40 mm de largo......................... 7

7. Tubo del perianto recto, hojas caducas en floración. Caliphruria

- Tubo del perianto encorvado, hojas persistentes durante la floración Eucharis 
8. Ovario ínfero

- Ovario súpero 15

9. Plantas con rizoma o raíces filamentosas. .10

- Plantas con bulbo. .11

10. Fruto cápsula, ovario trígono y alargado, tépalos amarillos, hojas $>80 \mathrm{~cm}$ de largo. Pamianthe

- Fruto en baya, ovario globoso, tépalos rojo-anaranjados, hojas $<60 \mathrm{~cm}$ de largo. Clivia

11.Estambres marcadamentes exsertos, tépalos blancos, ensiformes y patentes en la porción distal... Hymenocallis

- Estambres insertos, tépalos de diversos colores, nunca ensiformes. 12

12. Flor con paraperigonio, estaminal o no estaminal

- Flor carente de paraperigonio. 14

13. Paraperigonio no estaminal (corona), tépalos rojos, anaranjados o morados Hippeastrum

- Paraperigonio estaminal (copa), tépalos amarillos, blancos o rosados. Habranthus

14. Flores solitarias, hojas menos de $2 \mathrm{~cm}$ de ancho, tépalos de menos de $5 \mathrm{~cm}$ de largo........... Zephyranthes

- Inflorescencias multifloras, hojas más de $3 \mathrm{~cm}$ de ancho, tépalos de más de $5 \mathrm{~cm}$ de largo. Crinum

15.Hojas dísticas, plantas carentes de aroma...Agapanthus - Hojas espiraladas, plantas fuertemente aromáticas......16

16. Paraperigonio estaminal presente. Tulbaghia

- Paraperigonio estaminal ausente. ..17

17.Inflorescencia con menos de 25 flores.

Nothoscordum

- Inflorescencia esférica, con 50-500 flores. Allium

Subfamilia Agapanthoideae: Hierbas rizomatosas, con hojas dísticas, flores azules o blancas, ovario súpero.

\section{Agapanthus}

Hierbas perennes, 30-110 cm de altura. Rizoma reducido. Hojas 6-20, dísticas, sésiles, liguladas; lámina foliar linear, $20-70 \mathrm{~cm} \times 1.5-5.5 \mathrm{~cm}$. Inflorescencia umbelada; escapo afilo, 0.5-1 m de largo, con 2 brácteas distales unidas entre sí a lo largo de uno de sus lados. Flores numerosas (más de 100), actinomorfas o ligeramente zigomorfas, hermafroditas; perigonio campanulado o infundibuliforme; tépalos 6, libres en la parte distal, 40-50 $\mathrm{mm} \times 6-11 \mathrm{~mm}$, azules, ocasionalmente blancos, connados en la base; pedicelo 4-12 cm de largo. Estambres 6, libres; filamentos filiformes, insertos; anteras oblongas, dorsifijas, amarillas. Ovario súpero, trilocular; estigma capitado. Frutos cápsulas loculicidas. Semillas 20-100, planas, aladas, con testa negra brillante.

Una especie está presente en Colombia.

\subsection{Agapanthus praecox Willd.}

Introducida, cultivada. $1500-2600$ m s. n. m.

Distribución: originaria de Sudáfrica, cultivada ampliamente en diferentes regiones de Colombia.

Departamentos: Antioquia, Boyacá, Caldas, Cauca, Cundinamarca, Bolívar, Huila, Nariño, Quindío, Risaralda, Tolima y Valle del Cauca.

Usos: ornamental.

Ejemplar de referencia: R. Fonnegra 2632 (HUA).

Nombre vernáculo: agapanto.

Subfamilia Allioideae: Hierbas con aroma a cebolla o ajo, flores pequeñas, ovario súpero.

\section{Allium}

Hierbas perennes, $30-80 \mathrm{~cm}$ de altura. Bulbos tunicados, 1-15 cm de diámetro, solitarios o agrupados, con aroma a ajo o cebolla, con 1 a varias escamas. Hojas 1-12, arrosetadas, espiraladas, sésiles, liguladas, envainantes en la base, planas o cilíndricas, macizas o huecas, con aroma y sabor a ajo o cebolla; lámina foliar linear, filiforme o semicircular (fistulosa) en sección transversal, 1-60 cm x $0.2-8 \mathrm{~cm}$. Inflorescencia en umbela densa, esférica; esca- 
po 0.1-0.8 m de largo, con 1-3 brácteas connadas. Flores 50-500, actinomorfas; perigonio campanulado; tépalos libres, 6 en 2 verticilos de 3, marcescentes, angostamente lanceolados, 3-7 mm x 2-2.5 mm, blancos, rojos, rosados, lila, verdes o amarillos; pedicelo delgado, desarticulado y a menudo bracteolado, 1-50 mm de largo. Estambres 6, libres o fusionados en la base del perianto; filamentos exsertos o insertos, adnados en la base a los tépalos, a menudo 3-lobados; anteras, dorsifijas, introrsas, con dehiscencia longitudinal. Ovario súpero, trilocular; estigma entero a 3-lobado; estilo solitario, ginobásico. Frutos cápsulas loculicidas, globosas a ovoides. Semillas 4-6, subglobosas o aplanadas, pequeñas, con testa negra.

Cinco especies están presentes en Colombia.

\subsection{Allium ampeloprasum L.}

Introducida, cultivada. $2000-2800$ m s. n. m.

Distribución: originaria de Asia. Cultivada en Colombia, especialmente en la cordillera Oriental.

Departamentos: Boyacá, Cundinamarca y Nariño. Usos: comestible.

Ejemplar de referencia: A. Beltrán 291 (COL).

Nombres vernáculos: puerro, cebolla puerro.

\subsection{Allium cepa L.}

Introducida, cultivada. $1000-2700$ m s. n. m.

Distribución: originaria de Asia central. En Colombia cultivada en las áreas andinas de las tres cordilleras.

Departamentos: Antioquia, Caldas, Cauca, Cundinamarca, Nariño, Norte de Santander y Santander.

Usos: comestible, medicinal.

Ejemplar de referencia: J.I. Villa 9 (HUA).

Nombres vernáculos: cebolla cabezona, cebolla de huevo, cebolla blanca, cebolla morada, cebolla peruana, cebolla de a ciento, papa cebolla, cebolla.

\subsection{Allium fistulosum L.}

Introducida, cultivada. $1500-2700$ m s. n. m.

Distribución: originaria del sudeste de Asia y cultivada en todo el mundo. En Colombia se cultiva ampliamente en las tres cordilleras.
Departamentos: Antioquia, Boyacá, Cauca, Cundinamarca, Nariño, Norte de Santander, Risaralda, Santander y Valle del Cauca.

Usos: comestible.

Ejemplar de referencia: R. Londoño 360 (HUA, MEDEL). Nombres vernáculos: cebolla junca, cebolla larga, cebolla de rama, cebolla de rabo, cebolla junco, cebolla común, cebolla, cebollín.

\subsection{Allium satioum L.}

Introducida, cultivada. $1500-2600$ m s. n. m.

Distribución: originaria del Mediterráneo y en la actualidad cultivada en todo el mundo. En Colombia se cultiva ampliamente en la región Andina.

Departamentos: Antioquia, Boyacá, Caldas, Cauca, Cundinamarca, Nariño, Santander y Valle del Cauca.

Usos: comestible, medicinal.

Ejemplar de referencia: M. Escobar 5 (HUA).

Nombres vernáculos: ajo, ajo macho.

\subsection{Allium schoenoprasum L.}

Introducida, cultivada. $1800-2600$ m s. n. m.

Distribución: originaria de Siberia. Cultivada como planta ornamental en diferentes zonas de Colombia.

Departamentos: Antioquia, Boyacá, Cundinamarca y Putumayo.

Usos: ornamental.

Ejemplar de referencia: Corporación Ambiental UdeA 13 (HUA).

Nombres vernáculos: cebollín, cibulé.

\section{Nothoscordum}

Hierbas perennes, aromáticas, 20-60 cm. Bulbos con túnica gris a marrón, 1.4-2.3 cm de diámetro, compuestos por varias escamas. Hojas 2-10, arrosetadas, espiraladas, sésiles, glabras; lámina foliar linear, 20-45 cm x 0.3-1.2 $\mathrm{cm}$. Inflorescencia umbelada, rodeada por una espata; escapo 20-60 cm de largo, con 2 brácteas opuestas. Flores 6-25, actinomorfas, fragantes; perigonio infundibuliforme; tépalos unidos en la base, 6 en 2 verticilos, uninervios, 7-15 mm x 1.8-4.5 mm, blancos-amarillo, con costa lila o marrón; pedicelo 1.4-6 cm de largo. Estambres 6, 
libres; filamentos insertos, adnados en la parte inferior del perianto; anteras introrsas, dorsifijas, amarillas. Ovario súpero, sésil, trilocular; estilo filiforme; estigma entero a subcapitado. Frutos cápsulas loculicidas, ovoides, membranáceas. Semillas 24-36, esféricas, reducidas, con testa negra.

Una especie está presente en Colombia.

\subsection{Nothoscordum gracile (Aiton) Stearn}

Nativa, cultivada. 1000-2500 m s. n. m.

Distribución: descrita de propágulos provenientes de Jamaica. Conocida en Colombia sólo de poblaciones silvestres en el bosque húmedo premontano y montano bajo de Antioquia y cultivada en la cordillera Oriental.

Departamentos: Antioquia y Cundinamarca.

Usos: ornamental.

Ejemplar de referencia: J. Pérez 252 (MEDEL).

Nombres vernáculos: ninguno conocido.

\section{Tulbaghia}

Hierbas con olor a ajo o cebolla, $30-70 \mathrm{~cm}$ de altura. Rizomas fibrosos o bulbos de 1-2 cm de diámetro, con túnica membranosa, marrón. Hojas 4-10, arrosetadas, espiraladas, sésiles, con olor a ajo o cebolla, erectas, perennes; lámina foliar linear a ovada, carnosa, sésil, con ápice obtuso, $15-50 \mathrm{~cm} \times 0.3-1.9 \mathrm{~cm}$. Inflorescencia umbelada; escapo terete, $30-70 \mathrm{~cm}$ de largo, con 2 brácteas terminales. Flores 3-40, actinomorfas; perigonio infundibuliforme o hipocrateriforme; tépalos libres, 6 en 2 verticilos, menos de $2 \mathrm{~cm}$ de largo, violetas o blancos; pedicelo $1-5 \mathrm{~cm}$ de largo. Estambres 6, formando paraperigonio estaminal (copa) pequeño, traslúcido; anteras oblongas, dorsifijas, sésiles, en 2 verticilos. Ovario súpero, trilocular, sésil; estigma capitado. Frutos cápsulas dehiscentes, triloculares, rodeados por restos del perigonio. Semillas numerosas, angulosas o aplanadas, con testa negra.

Dos especies están presentes en Colombia.

\subsection{Tulbaghia alliacea L. f.}

Introducida, cultivada. $2000-2600$ m s. n. m.
Distribución: descrita originalmente de Sudáfrica, cultivada como planta ornamental.

Departamentos: Antioquia, Caldas y Cundinamarca. Usos: ornamental y medicinal.

Ejemplar de referencia: Y. Pérez 001 (COL).

Nombres vernáculos: cebolleta.

\subsection{Tulbaghia violacea Harv.}

Introducida, cultivada. $1200-2800$ m s. n. m.

Distribución: descrita de Sudáfrica y cultivada como planta ornamental en diferentes países y en diferentes zonas de Colombia.

Departamentos: Antioquia, Cundinamarca, Quindío y Valle del Cauca.

Usos: ornamental y medicinal.

Ejemplar de referencia: M. Hernández 1401 (COL). Nombres vernáculos: cebolleta, cebollina.

Subfamilia Amaryllidoideae: Hierbas con flores grandes y llamativas, ovario ínfero.

\section{5. $x$ Calicharis Meerow}

Hierbas perennes, hasta $60 \mathrm{~cm}$ de altura. Bulbo con túnica marrón, 3.8-5 cm de diámetro, cuello corto y delgado. Hojas 2-3, arrosetadas, espiraladas, pecioladas, perennes; lámina foliar elíptica, $21-35 \mathrm{~cm} \times 10.5-15 \mathrm{~cm}$, plegada. Inflorescencia umbelada; escapo 50-60 cm de largo y 4-5.5 mm de diámetro. Flores 4-7, actinomorfas, aromáticas, perigonio hexámero infundibuliforme-campanulado, dilatándose gradualmente hasta la mitad de la longitud del tubo, luego dilatado abruptamente; tépalos 6, libres, agudo-apiculados u obtusos, fusionados en la base, $19-28 \mathrm{~mm}$ x 12-25 mm, blancos, excepto la mayoría del tubo verde; pedicelo 4.5-5 mm de largo. Estambres 6, formando paraperigonio estaminal (copa) edentado o raramente con 1 diente pequeño; filamentos insertos, anteras linear-oblongas, en su mayoría sin polen. Ovario ínfero, globoso-elipsoide, trilocular. Frutos y semillas no conocidos.

Nota: Este es un híbrido intergenérico generado por Caliphruria subedentata Baker y Eucharis sanderi Baker, según Meerow (1989).

Una especie presente en Colombia 


\section{1. x Calicharis butcheri (Traub) Meerow}

Nativa. 200-1000 m s. n. m.

Distribución: descrita a partir de colecciones realizadas en Panamá y el Valle del Cauca. En Colombia se encuentra en bosques húmedos tropicales del sur de la cordillera Occidental. Las colecciones de este híbrido son muy escasas en Colombia.

Departamentos: Cauca y Valle del Cauca. Usos: ninguno conocido.

Ejemplar de referencia: F. K. Lehmann 2736 (BM, K). Nombres vernáculos: ninguno conocido.

\section{Caliphruria}

Hierbas perennes, hasta $46 \mathrm{~cm}$ de altura. Bulbo con túnica marrón, $3 \mathrm{~cm}$ de diámetro, a menudo articulado en el ápice en un cuello delgado. Hojas 2-4, arrosetadas, espiraladas, pecioladas, liguladas, ausentes en la floración; lámina foliar ovada, ovado-elíptica o elíptica, 10 $20 \mathrm{~cm} \times 3.8-6.3 \mathrm{~cm}$. Inflorescencia umbelada; bractéolas presentes; escapo 25-45 cm de largo y 2-5.5 mm de diámetro, con 2 brácteas amplias. Flores 5-10, actinomorfas, aromáticas; perigonio hexámero infundibuliforme; tubo del perianto recto, dilatándose gradualmente desde la base; tépalos 6, libres, elípticos u ovados, apiculados, obtusos, fusionados en la base, 13.5-20 mm x 7-10 $\mathrm{mm}$, blancos, excepto en la parte distal-media del tubo; pedicelo 1-4 cm de largo. Estambres 6, formando paraperigonio estaminal (copa) edentado, raramente con 1-2 dientes pequeños o con dientes mucho más largos que los filamentos; filamentos insertos, anteras lineares. Ovario ínfero, globoso-elipsoide, trilocular. Frutos cápsulas. Semillas 1-2 por lóculo, elipsoidales, testa negra o marrón.

Tres especies presentes en Colombia.

\subsection{Caliphruria hartwegiana Herb.}

Endémica. 600-2500 m s. n. m.

Distribución: descrita de especímenes recolectados en cercanías a Guaduas, Cundinamarca. Crece en bosques tropicales y premontanos de la parte central y sur de la cordillera Oriental, incluyendo el valle del río Magdalena.
Departamentos: Cundinamarca y Huila.

Usos: ornamental.

Ejemplar de referencia: J. Torres 1241 (COL).

Nombres vernáculos: ninguno conocido.

\subsection{Caliphruria subedentata Baker.}

Endémica. 600-2500 m s. n. m.

Distribución: descrita a partir de especímenes cultivados en $\mathrm{K}$, provenientes de una zona no determinada de Colombia. Conocida de colecciones del sur de las cordilleras Occidental y Central, y centro de la cordillera Oriental. Especie poco frecuente, que crece en bosques tropicales y premontanos.

Departamentos: Cauca, Cundinamarca, Huila y Valle del Cauca.

Usos: ornamental.

Ejemplar de referencia: A. Fernández 2795 (COL).

Nombres vernáculos: billete, cebolla, cebolleta blanca, cebollina, azucena, lirio, estrella de Belén, estrella del Caquetá, reina del Caquetá, varita de san José, vida del hombre.

\subsection{Caliphruria tenera Baker}

Endémica. 400-1100 m s. n. m.

Distribución: descrita de colecciones del valle del río Magdalena. Crece en el bosque tropical del sur de la cordillera Central y en la parte central de la cordillera Oriental. Es muy poco frecuente y se conoce de muy pocas localidades.

Departamentos: Cundinamarca, Huila, Tolima.

Usos: ornamental.

Ejemplar de referencia: P. Silverstone-Sopkin 11957 (CUVC).

Nombres vernáculos: ninguno conocido.

\section{Clivia}

Hierbas perennes, 0.2-0.6 m de altura. Raíces rizomatosas, carentes de bulbos, fasciculadas, fibrosas. Hojas 14-20, dísticas, sésiles, perennes, coriáceas, lustrosas; lámina foliar linear, $45-60 \mathrm{~cm} \times 3-5 \mathrm{~cm}$. Inflorescencia umbelada; escapo $30.5-45.7 \mathrm{~cm}$ de largo con varias brácteas. Flores 10-50, actinomorfas o ligeramente zigomorfas, hermafroditas, aromáticas; perigonio hexámero infundibuliforme, fusionado 
en la base; tépalos 6, libres, $5-7.5 \mathrm{~cm} \times 2.5 \mathrm{~cm}$, anaranjados a rojizos; pedicelo $2-5 \mathrm{~cm}$. Estambres 6, libres; filamentos cortos, glabros, insertos; anteras oblongas, dorsifijas, amarillas. Ovario ínfero, trilocular, globoso; estigma subtrilobado a trilobado; estilo filiforme. Frutos bayas, globosas, rojas-anaranjadas. Semillas 1-15, globosas, bulbiformes, carentes de epispermo.

Una especie está presente en Colombia.

\subsection{Clivia miniata Regel.}

Introducida, cultivada. $1500-2600$ m s. n. m.

Distribución: descrita de Sudáfrica, se cultiva como ornamental en casi todo el mundo.

Departamentos: Antioquia, Boyacá, Caldas, Cauca, Cundinamarca, Quindío, Risaralda, Santander y Valle del Cauca. Usos: ornamental.

Ejemplar de deferencia: F. Alzate 5215 (HUA). Nombres vernáculos: clivia, doce apóstoles, tulipán.

\section{Crinum}

Hierbas perennes, $0.6-1.5 \mathrm{~m}$ de altura. Bulbos blancos, con túnica marrón, voluminosos, $6-30 \mathrm{~cm}$ de diámetro. Hojas, 6-15, arrosetadas, espiraladas, (dísticas en especies que no se encuentran en Colombia), sésiles, liguladas; lámina foliar linear a lanceolada, larga, plana, $30-150 \mathrm{~cm} \mathrm{x}$ 3-12 cm. Inflorescencia umbelada, o flor solitaria; escapo terete, macizo, 0.3-1.5 m de largo, con 2 o más brácteas estrechamente triangulares. Flores numerosas 7-100, ocasionalmente 1 (no en Colombia), actinomorfas o zigomorfas, hermafroditas, aromáticas; perigonio campanulado, infundibuliforme, hipocrateriforme o rotado, tubo del perianto largo; tépalos 6, fusionados en la base, 5-18 $\mathrm{cm} \times 1-1.7 \mathrm{~cm}$, blancos, verdes, rosados o rojos, vistosos; pedicelo 1-12 cm de largo. Estambres 6, libres; filamentos blancos, insertos; anteras dorsifijas, marrones. Ovario ínfero, trilocular; estigma subcapitado. Frutos cápsulas dehiscentes, globosas. Semillas numerosas, verrugosas, aplanadas o voluminosas, testa blanca, verde a marrón.

Crinum es el género más ampliamente distribuido de la familia, encontrándose en América, África y Asia (Strydom, 2005).
Ocho especies están presentes en Colombia.

\subsection{Crinum $\mathrm{x}$ amabile Donn}

Introducida, cultivada. $800-1000$ m s. n. m.

Distribución: originaria de la India. Cultivada y solo conocida de colecciones realizadas en la ciudad de Cali. Departamentos: Valle del Cauca.

Usos: ornamental.

Ejemplar de referencia: P. Silverstone-Sopkin 11203 (CUVC, MO).

Nombres vernáculos: ninguno conocido.

Nota: híbrido de Crinum asiaticum y Crinum zeylanicum.

8.2. Crinum bulbispermum (Burm.f.) Milne-Redh. \&Schweick

Introducida, cultivada. 0-2700 m s. n. m.

Distribución: descrita de Sudáfrica y cultivada como ornamental en diferentes países.

Departamentos: Boyacá, Cundinamarca y San Andrés. Usos: ornamental.

Ejemplar de referencia: F. Alzate 4334 (HUA).

Nombres vernáculos: lirio.

\subsection{Crinum erubescens Aiton.}

Nativa, cultivada. 0-500 m s. n. m.

Distribución: originaria de Centroamérica y Suramérica; crece en bosques húmedos tropicales de la Amazonia, Orinoquia, Chocó biogeográfico y el Caribe colombiano

Departamentos: Amazonas, Arauca, Chocó, Cauca, Córdoba, Nariño y Valle del Cauca.

Usos: ninguno conocido.

Ejemplar de referencia: $R$. Vásquez 12480 (MO).

Nombres vernáculos: lirio, tulipán.

\subsection{Crinum graciliflorum Kunth \& C.D. Bouché}

Nativa. 0-500 m s. n. m.

Distribución: descrita de Venezuela; en Colombia se encuentra en el Chocó biogeográfico y piedemonte occidental de la cordillera Occidental, prosperando en bosques húmedos tropicales.

Departamentos: Chocó, Cauca y Valle del Cauca. 
Usos: ninguno conocido.

Ejemplar de referencia: $B$. Latorre 12 (CAUP).

Nombres vernáculos: lirio.

8.5. Crinum jagus (J. Thomps.) Dandy.

Introducida y cultivada. 0-2200 m s. n. m.

Distribución: descrita del centro de África y cultivada en diferentes países. En Colombia se cultiva como ornamental. Departamentos: Antioquia, Boyacá, Cauca, Cundinamarca, Quindío, Santander y Valle del Cauca.

Usos: ornamental.

Ejemplar de referencia: F. Alzate 5270 (HUA).

Nombres vernáculos: lirio.

\subsection{Crinum kunthianum M. Roem.}

Nativa, cultivada. 0-1200 m s. n. m.

Distribución: descrita a partir de una colección realizada en Turbaco, Bolívar. Crece también en Chocó biogeográfico y zonas circundantes al golfo de Urabá.

Departamentos: Antioquia, Bolívar, Cauca, Chocó, Nariño y Valle del Cauca.

Usos: ornamental.

Ejemplar de referencia: E. Rentería et al. 5311 (HUA). Nombres vernáculos: lirio, lirio blanco.

8.7. Crinum moorei Hook. f.

Introducida, Cultivada. $1400-2600$ m s. n. m.

Distribución: descrita de Sudáfrica. Se cultiva en casi todo el mundo como ornamental.

Departamentos: Antioquia y Cundinamarca.

Usos: ornamental.

Ejemplar de referencia: J. Duque-Jaramillo 3132 (COL). Nombres vernáculos: lirio.

\subsection{Crinum zeylanicum (L.) L.}

Introducida, cultivada. 200-1000 m s. n. m.

Distribución: originaria de Asia tropical. En Colombia es cultivada en diversas regiones del piso altitudinal tropical. Departamentos: Boyacá, Cauca, Santander y Valle del Cauca.

Usos: ornamental.
Ejemplar de referencia: F. Alzate 4339 (HUA).

Nombres vernáculos: lirio.

\section{Eucharis}

Hierbas perennes, que alcanzan $45-61 \mathrm{~cm}$ de altura. Bulbos con túnica marrón, 2-8 cm de diámetro, subglobosos. Hojas 1-2 (-4), permaneciendo durante la floración, arrosetadas, espiraladas, pecioladas, carentes de lígula, ligeramente glaucas en una especie; lámina foliar elíptica, lanceolada u ovada, $15-55 \mathrm{~cm}$ x 6-21 cm. Inflorescencia umbelada; escapo afilo, terete, $21-80 \mathrm{~cm}$ de largo, con 3 brácteas ovadas, 3-4 cm de largo. Flores 2-12, actinomorfas, tubulares, aromáticas; perigonio crateriforme, campanulado o infundibuliforme, tubo del perianto encorvado; tépalos 6, libres en la porción distal, fusionados en la base, $2.0-3.5 \mathrm{~cm} \times 0.8-1.4 \mathrm{~cm}$, blancos; pedicelo 1.5-2.5 cm de largo. Estambres 6, formando paraperigonio estaminal (copa) blanco, sin dientes o con dos dientes entre cada par de estambres de 12-15 mm de longitud, filamentosos; filamentos insertos; anteras lineares, basifijas o dorsifijas. Ovario ínfero; estigma trilobulado; estilo filiforme. Frutos cápsulas loculicidas, 1-2.5 cm de diámetro. Semillas 3-37, elipsoidales, globosas u ovoides, con testa anaranjada, negra o azul.

Ocho especies están presentes en Colombia.

\subsection{Eucharis bonplandii (Kunth) Traub.}

Endémica. 200-1300 m s. n. m.

Distribución: descrita a partir de colecciones de Cundinamarca. Al igual que todas las especies del género, este taxón es poco frecuente, pero es una de las más abundantes a nivel local al interior de bosques tropicales y premontanos de las tres cordilleras andinas.

Departamentos: Caldas, Cundinamarca, Meta, Quindío, Risaralda, Santander, Tolima y Valle del Cauca.

Usos: no conocido.

Ejemplar de referencia: F. Alzate 5107 (HUA).

Nombres vernáculos: ninguno conocido.

9.2. Eucharis candida Planch. \& Linden.

Nativa. $100-1100$ m s. n. m. 
Distribución: descrita de especímenes cultivados en Francia, a partir de propágulos provenientes de Colombia, sin que se tenga certeza de la localidad exacta de origen. Crece también en Ecuador y Perú; en Colombia es poco frecuente y sólo se conoce de colecciones realizadas en bosques tropicales de la Amazonia y la Orinoquia.

Departamentos: Amazonas, Meta y Putumayo. Usos: ninguno conocido.

Ejemplar de referencia: G. Lozano 594 (COL). Nombres vernáculos: cebollita.

\subsection{Eucharis caucana Meerow.}

Endémica.100-1200 m s. n. m.

Distribución: descrita a partir de colecciones del Valle del Cauca. Especie rara, sólo se conoce de escasas localidades en el valle del río Cauca, la cordillera Occidental y Chocó biogeográfico. Considerada en grave peligro de extinción. Departamentos: Chocó, Quindío, Risaralda y Valle del Cauca.

Usos: ninguno conocido.

Ejemplar de referencia: P. Silverstone-Sopkin 3064 (CUVC). Nombres vernáculos: ninguno conocido.

\subsection{Eucharis formosa Meerow.}

Nativa, cultivada. $100-1800$ m s. n. m.

Distribución: descrita del piedemonte amazónico ecuatoriano. En Colombia se encuentra silvestre en los bosques tropicales de la Amazonia y es cultivada como planta ornamental en algunas regiones del país.

Departamentos: Amazonas, Caquetá, Cauca y Putumayo. Usos: ornamental.

Ejemplar de referencia: M. Mera 01 (CAUP).

Nombres vernáculos: sachacebolleta, ajo de perdiz.

\subsection{Eucharis $x$ grandiflora Planch. \& Linden}

Nativa, cultivada.100-1500 m s. n. m.

Distribución: descrita a partir de colecciones realizadas en el Chocó y cultivada en Bélgica. Se encuentra en bosques tropicales y premontanos de la cordillera Occidental. Departamentos: Risaralda y Valle del Cauca.

Usos: ninguno conocido.
Ejemplar de referencia: J. Ramos 1898 (CUVC).

Nombres vernáculos: ninguno conocido.

\subsection{Eucharis lehmannii Regel}

Endémica. 1200 m s. n. m.

Distribución: descrita a partir de material recolectado cerca de Popayán y propagado en Bruselas por J. Linden. Sólo conocida del sur de la cordillera Occidental.

Departamentos: Cauca.

Usos: ninguno conocido.

Ejemplar de referencia: F. K. Lehmann 5883 (K).

Nombres vernáculos: ninguno conocido.

\subsection{Eucharis sanderi Baker.}

Nativa. 0-1000 m s. n. m.

Distribución: descrita con base en especímenes cultivados en Inglaterra a partir de bulbos recolectados en Colombia, sin que se conozca su exacta localidad. Con escasas poblaciones, en bosques húmedos tropicales de la cordillera Occidental y Chocó biogeográfico.

Departamentos: Caldas, Cauca, Chocó, Nariño, Risaralda y Valle del Cauca.

Usos: ninguno conocido.

Ejemplar de referencia: A. Yusti-Muñoz 107 (CUVC). Nombres vernáculos: ninguno conocido.

\subsection{Eucharis ulei Kraenzl.}

Nativa. $100-300$ m s. n. m.

Distribución: descrita de Brasil, endémica de los bosques húmedos tropicales de la cuenca amazónica. En Colombia solo se conoce de especímenes recolectados en cercanías a Leticia, departamento de Amazonas.

Departamentos: Amazonas.

Usos: ninguno conocido.

Ejemplar de referencia: A. Rudas 3438 (MO).

Nombres vernáculos: ninguno conocido.

\section{Habranthus}

Hierbas perennes, $15.2-40 \mathrm{~cm}$ de altura. Bulbos $3.5-5 \mathrm{~cm}$ de diámetro, ovoides o globosos, con túnica de color marrón o negro. Hojas arrosetadas, espiraladas, sésiles, liguladas, 
deciduas; lámina foliar linear, $30-40 \mathrm{~cm} \times 0.4-2 \mathrm{~cm}$. Inflorescencia umbelada o reducida a una sola flor; bractéolas presentes; escapos huecos, 15-30 cm de largo, con 1 bráctea. Flores 1-4, ligeramente zigomorfas, suberectas; perigonio crateriforme o infundibuliforme; tépalos 6, libres, 3-7 cm de largo, amarillos, blancos o rosados; pedicelo $3.3-6.5 \mathrm{~cm}$ de largo. Estambres 6, formando paraperigonio estaminal (copa) tubular, filamentosos, desiguales en longitud; filamentos filiformes, distalmente curvados, insertos; anteras dorsifijas, lineares u oblongas, amarillas. Ovario ínfero; estigma trilobado; estilo filiforme. Frutos cápsulas triloculares, subglobosas u oblongas; pericarpio delgado. Semillas numerosas, aladas, con testa marrón o negra.

Una especie se cultiva en Colombia.

\subsection{Habranthus robustus Herb.}

Introducida, cultivada. $1000-2000$ m s. n. m.

Distribución: descrita a partir de material proveniente de Argentina y propagado en Inglaterra. En Colombia se cultiva como ornamental.

Departamentos: Antioquia y Cundinamarca.

Usos: ornamental.

Ejemplar de referencia: $R$. Fonnegra 5424 (HUA, MO). Nombres vernáculos: cebolleta

\section{Hippeastrum}

Hierbas perennes, $30-80 \mathrm{~cm}$ de altura. Bulbos de $4-12 \mathrm{~cm}$ de diámetro, con túnica marrón a gris. Hojas 2-8, arrosetadas, espiraladas, sésiles, liguladas, apareciendo durante la floración; lámina foliar linear a lanceolada, plana, 20$90 \mathrm{~cm}$ x 2-5 cm. Inflorescencia umbelada; escapo hueco, $25-100 \mathrm{~cm}$ de longitud con 2 brácteas lanceoladas, verdes y marrón, 3-5 cm de longitud. Flores 2-14, zigomorfas, tubulares; perigonio infundibuliforme, tubo del perianto corto; tépalos 6, fusionados en la base, 6-15 cm x 3-5 $\mathrm{cm}$, rojos, anaranjados o morados, en ocasiones con paraperigonio no estaminal (corona) rudimentario, escamoso, poco visible; pedicelo 2.5-7 cm de largo. Estambres 6, libres; filamentos filiformes, insertos; anteras dorsifijas. Ovario ínfero, trilocular; estigma trilobado. Frutos cápsulas loculicidas, hasta de $2.5 \mathrm{~cm}$ de diámetro. Semillas numerosas, aplanadas con testa negra o marrón.
Cuatro especies están presentes en Colombia.

\subsection{Hippeastrum andreanum Baker.}

Endémica. 2000-2800 m s. n. m.

Distribución: descrita a partir de bulbos recolectados en la cordillera Central de Colombia y cultivados en Inglaterra. Se conoce de localidades disyuntas de las cordilleras Occidental y Oriental, poco frecuente.

Departamentos: Boyacá, Cauca y Meta.

Usos: ninguno conocido.

Ejemplar de referencia: L. Uribe 5191 (COL).

Nombres vernáculos: lirio.

\subsection{Hippeastrum barbatum Herb.}

Introducida, cultivada. $1000-2000$ m s. n. m.

Distribución: originaria de Sudáfrica y naturalizada en América. Cultivada en Colombia en la cordillera Oriental.

Departamentos: Boyacá, Cundinamarca.

Usos: ornamental.

Ejemplar de referencia: F. Alzate 4264 (HUA).

Nombres vernáculos: lirio.

Nota: en algunos tratamientos ha sido propuesto sinonimizar esta especie bajo Hippeastrum puniceum subsp. puniceum; sin embargo, no se presenta ninguna justificación para tomar dicha decisión

\subsection{Hippeastrum elegans (Spreng.) H.E. Moore}

Nativa. 500-1800 m s. n. m.

Distribución: especie de amplia distribución, desde Costa Rica hasta Brasil. En Colombia se encuentra en la Orinoquia, sabanas del Caribe y cordillera Oriental. Departamentos: Casanare, Cundinamarca, Magdalena, Meta y Tolima.

Usos: no conocido.

Ejemplar de referencia: F. Alzate 5175 (HUA). Nombres vernáculos: lirio.

\subsection{Hippeastrum puniceum (Lam.) Voss}

Nativa, cultivada. 100-3500 m s. n. m.

Distribución: originaria de Suramérica, descrita partir de material aparentemente procedente de una localidad 
del trópico de Suramérica. Cultivada en amplias zonas del continente. En Colombia se tienen registros para las tres cordilleras, Amazonia y Orinoquia, creciendo en un amplio rango altitudinal.

Departamentos: Amazonas, Antioquia, Boyacá, Casanare, Cundinamarca, Meta, Risaralda, Santander, Tolima y Valle del Cauca.

Usos: ornamental.

Ejemplar de referencia: J. Fernández-Alonso 21241 (COL). Nombres vernáculos: lirio, lirio rojo, azucena roja, cebolleta colorada, lirio rosado.

Nota: no se tiene claridad del origen de esta especie, ya que fue descrita a partir de especímenes cultivados en Europa y cuya procedencia no es clara. Sin embargo, el género $\mathrm{Hi}$ ppeastrum es de distribución restringida a América.

\section{Hymenocallis}

Hierbas perennes, $60-80 \mathrm{~cm}$ de altura. Bulbo $5-10 \mathrm{~cm}$ de diámetro, ovoide o globoso, con túnica fibrosa. Hojas 2-100, arrosetadas, espiraladas, sésiles, o rara vez pecioladas, liguliformes, perennes o caducas; lámina foliar linear a lanceolada, plana, $30-80 \mathrm{~cm} \times$ 2-8 cm. Inflorescencia umbelada o reducida a una sola flor; a menudo con bractéolas; escapo macizo, 0.3-1.2 m de largo, con 2-4 brácteas, ovadas, lanceoladas o triangulares, $5-7 \mathrm{~cm}$ de longitud. Flores 1-17, actinomorfas, hermafroditas, aromáticas, vistosas, ocasionalmente sésiles; perigonio infundibuliforme o hipocrateriforme, estrellado; tépalos 6, fusionados en la base, recurvados, en la parte distal ensiformes y angostos, patentes, 7-12 cm de largo, blancos; pedicelo 10-25 $\mathrm{cm}$ de largo. Estambres 6, formando un amplio paraperigonio estaminal (copa), membranoso; filamentos filiformes, marcadamente exsertos; anteras dorsifijas. Ovario ínfero, trilocular; estigma capitado a trilobado; estilo filiforme, terminal, exserto. Frutos cápsulas dehiscentes, subglobosas o elongadas, verdes, triloculares, carnosas. Semilla usualmente solitaria, angular, elipsoidal, carnosa, con testa negra o verde.

Tres especies están presentes en Colombia.

\subsection{Hymenocallis acutifolia (Herb.) Sweet}

Introducida. 1000-1500 m s. n. m.
Distribución: descrita de México, conocida en Colombia solamente de individuos cultivados en la zona urbana de Cali. Departamentos: Valle del Cauca.

Usos: ornamental.

Ejemplar de referencia: F. Cabezas s.n. (CUVC).

Nombres vernáculos: lirio.

12.2. Hymenocallis caribaea (L.) Herb.

Introducida. $1000-2000 \mathrm{~m} \mathrm{s.} \mathrm{n.} \mathrm{m.}$

Distribución: originaria del Caribe, descrita a partir de colecciones realizadas en Jamaica. Cultivada en Colombia en la cordillera Oriental.

Departamentos: Santander.

Usos: ornamental.

Ejemplar de referencia: F. Alzate 4337 (HUA).

Nombres vernáculos: ninguno conocido.

12.3. Hymenocallis littoralis (Jacq.) Salisb.

Nativa. 0-400 m s. n. m.

Distribución: descrita a partir de colecciones realizadas en Cartagena. Crece silvestre desde México hasta Perú. En Colombia se encuentra en las sabanas y las islas del Caribe, y Chocó biogeográfico.

Departamentos: Bolívar, Chocó, Córdoba, La Guajira, San Andrés y Providencia

Usos: ninguno conocido.

Ejemplar de referencia: $H$. Cuadros 3031 (MO).

Nombres vernáculos: lirio, cebolleta, cebolleta blanca, cebollina, azucena, azucena chirosa, chirosa, sirena, vara de san José, varita de san José, flor de pila.

\section{Narcissus}

Hierbas perennes, 5-80 $\mathrm{cm}$ de altura. Bulbo hasta de 4 cm de diámetro, tunicado. Hojas 2-5, arrosetadas, espiraladas, pecioladas, liguladas, glabras; lámina foliar linear, $8-50 \mathrm{~cm} \times 0.1-1.5 \mathrm{~cm}$. Inflorescencia umbelada o flor solitaria; escapo hueco, $12-80 \mathrm{~cm}$ de largo, con 1 bráctea membranosa. Flores 1-20, zigomorfas, aromáticas; tépalos internos formando corona campanulada, paraperigonial, no estaminal, muy desarrollada, amarilla, blanca, roja o verde, tépalos externos 6 , patentes, $2.5-15 \times 1.5-15 \mathrm{~mm}$, blancos o amarillos; pedi- 
celo 0.3-3 cm de largo. Estambres 6, libres; filamentos insertos; anteras introrsas. Ovario ínfero, trilocular; estigma alargado, no capitado; estilo inserto. Frutos cápsulas, subesféricas. Semillas numerosas, globosas, ovadas, con testa negra.

Una especie se cultiva en Colombia.

\subsection{Narcissus pseudonarcissus L.}

Introducida. 2000-2900 m s. n. m.

Distribución: originaria de Europa. Cultivada como ornamental.

Departamentos: Boyacá, Cauca y Nariño.

Usos: ornamental.

Ejemplar de referencia: B. Ramírez 22249 (CAUP).

Nombres vernáculos: narciso.

\section{Pamianthe}

Hierbas perennes. Raíces filamentosas, gruesas, que emergen de un pseudotallo. Hojas numerosas, dísticas, sésiles, glabras, liguliformes, ápice agudo, margen entero, nervio central conspicuo, 82.7-104.5 x 5.5-6.3 $\mathrm{cm}$. Inflorescencia pseudo-umbela, con flores orientadas en ángulo recto desde el ápice del pedicelo; escapo cilíndrico, brácteas caducas. Flores 9-10, con perigonio crateriforme, casi obsoleto; tépalos 6, libres, glabros, ovados, $2.8 \times 2.1 \mathrm{~cm}$, amarillos, ápice redondeado, engrosado; tépalos externos valvados; pedicelo $7-9 \mathrm{~cm}$ de largo. Estambres 6, fusionados en la base del perianto, formando paraperigonio estaminal (copa); filamentos insertos, adnados en el borde del paraperigonio estaminal, amarillos; anteras agrupadas en el centro de la flor, dorsifijas, versátiles, con dehiscencia longitudinal. Ovario ínfero, trilocular, trígono, alargado; estigma trilobado, con lóbulos papilados; estilo curvo. Frutos cápsulas dehiscentes, 3 valvas elípticas a obovadas, glabras, lisas. Semillas hasta 233, aladas, glabras, falciformes, testa marrón oscuro, alas delgadas, membranosas, marrón claro.

Una especie está presente en Colombia.
14.1. Pamianthe ecollis Silverst., Meerow \& Sánchez-Taborda

Endémica. 2839 m s. n. m.

Distribución: recientemente descrita a partir de especímenes encontrados en el norte de la Serranía El Pinche, en la vertiente occidental de la cordillera Occidental de Colombia. Crece en bosques de niebla áreas abiertas en taludes empinados cerca de arroyos o cuerpos de agua y sobre sustratos rocosos.

Departamentos: Cauca.

Usos: ninguno conocido.

Ejemplar de referencia: J. A. Sánchez-Taborda 2870 (CUVC, CAUP).

Nombres vernáculos: ninguno conocido.

\section{Phaedranassa.}

Hierbas perennes, hasta $60 \mathrm{~cm}$ de alto. Bulbo de 2-6 cm de diámetro, globoso, con túnica crema a marrón. Hojas 1-3, arrosetadas, espiraladas, pecioladas, hasta $10 \mathrm{~cm}$ de largo, carentes de lígula, caducas en la antesis; lámina foliar linear, lanceolada o estrechamente elíptica, $20-40 \mathrm{~cm} \times 2-7.5 \mathrm{~cm}$. Inflorescencia umbelada; bractéolas marcescentes; escapo $50-85 \mathrm{~cm}$ de largo, con varias brácteas, lineares, ovadas, lanceoladas, oblongas o triangulares a filamentosas. Flores 4-15, actinomorfas, péndulas; perigonio tubular; tépalos 6, en dos verticilos, fusionados en la base, 3-6 cm de largo, rosados-rojos y amarillos o verdes en la porción distal, con variegaciones verdes y con una franja delgada, amarilla cerca del ovario; pediceladas. Estambres 6, libres; filamentos rosados, marcadamente exsertos; anteras dorsifijas, amarillas. Ovario ínfero, trilocular, globoso; estilo filiforme. Frutos cápsulas dehiscentes, angulosas. Semillas numerosas, aplanadas, con testa negra, lustrosa.

Dos especies están presentes en Colombia.

15.1. Phaedranassa dubia (Kunth) J.F. Macbr.

Nativa. 900-2900 m s. n. m. 
Distribución: descrita a partir de Quito, Ecuador. En Colombia crece en áreas de bosque premontano y montano bajo del sur de la cordillera Occidental y Nudo de los Pastos. Departamentos: Cauca y Nariño.

Usos: ninguno conocido.

Ejemplar de referencia: C. Ariza 429 (COL).

Nombres vernáculos: ninguno conocido.

Nota: algunos especímenes de Phaedranassa recolectados en los departamentos de Cauca y Nariño han sido determinados como $P$. ventricosa; sin embargo, no se advierten diferencias morfológicas con el concepto taxonómico de $P$. dubia. Considerando esto y el sobrelapamiento de las áreas de distribución de los especímenes determinados con ambos nombres, se mantiene $P$. dubia, especie publicada con antelación. El género Phaedranassa amerita una revisión taxonómica.

\subsection{Phaedranassa lehmannii Regel}

Nativa. 500-1500 m s. n. m.

Distribución: frecuente, descrita a partir de colecciones del Cauca. Se distribuye en bosques tropicales y premontanos del sur de la cordillera Occidental y Nudo de los Pastos, llegando hasta el centro de Ecuador.

Departamentos: Cauca, Nariño y Valle del Cauca. Usos: ninguno conocido.

Ejemplar de referencia: F. Alzate 5106 (HUA).

Nombres vernáculos: ninguno conocido.

\section{Plagiolirion}

Hierbas perennes. Bulbos $3.4 \mathrm{~cm}$ de diámetro, con túnica marrón. Hojas 2-3, arrosetadas, espiraladas, pecioladas, carentes de lígula; lámina foliar elíptica u oblonga, plana. Inflorescencia umbelada; escapo macizo, hasta $16 \mathrm{~cm}$ de largo, con 2 brácteas marcescentes, lineares $o$ lanceoladas. Flores 8-26, zigomorfas, carentes de aroma; tépalos 6, libres, lanceolados, de $3 \mathrm{~cm}$ de largo, color blanco; pedicelo hasta $2 \mathrm{~cm}$ de largo. Estambres 6, formando paraperigonio estaminal (copa) con pequeños dientes lanceolados entre los filamentos filiformes, exsertos o insertos, monadelfos; anteras amarillas o blancas. Ovario ínfero, elipsoidal, con tres lóculos. Frutos cápsulas loculicidas. Semillas 1-3, elipsoidales, con testa negra brillante.

Una especie está presente en Colombia.

\subsection{Plagiolirion horsmannii Baker}

Endémica. 900-2000 m s. n. m.

Distribución: descrita a partir de individuos cultivados en Inglaterra mediante bulbos provenientes del Valle del Cauca. Solo se tienen registros para tres localidades de la parte centro-norte de la cordillera Central y la parte media del Valle del río Cauca, creciendo en bosques húmedos premontano, hasta secos tropicales.

Departamentos: Antioquia, Risaralda y Valle del Cauca. Usos: ninguno conocido.

Ejemplar de referencia: P. Silverstone-Sopkin 7236 (CUVC). Nombres vernáculos: ninguno conocido.

\section{Scadoxus}

Hierbas perennes o caducas, 0.2-1.2 m de altura. Raíces rizomatosas o bulbos de $5 \mathrm{~cm}$ de diámetro. Hojas hasta 9, arrosetadas, espiraladas, pecioladas, formando un pseudotallo, con una vena media prominente, largas; lámina foliar elíptica-lanceolada, las márgenes tienden a ser onduladas, $20-35 \mathrm{~cm} \times 8-15 \mathrm{~cm}$. Inflorescencia umbelada formando una esfera densa; escapo terete, 12-110 cm largo, con 4 o más brácteas grandes, libres o fusionadas. Flores 10-200, zigomorfas, pedunculadas; perigonio tubular; tépalos 6 en 2 verticilos, fusionados en la base, 15-35 mm de largo, de color anaranjado, rojo o rosado; pedicelo 15-45 mm de largo. Estambres 6, libres; filamentos rojos, filiformes, exsertos; anteras amarillas. Ovario ínfero; estigma capitado; estilo filamentoso. Frutos bayas verdes, y rojas a anaranjadas al madurar, globosas. Semillas 1-3, aplanadas, carnosas, testa pálida.

Una especie se cultiva en Colombia.

17.1. Scadoxus multiflorus (Martyn) Raf., Fl. Tellur. 4: 19. 1838.

Introducida, cultivada. $2000-2600$ m s. n. m. 
Distribución: originaria de África subsahariana. En Colombia se cultiva en la sabana de Bogotá.

Departamentos: Cundinamarca.

Usos: ornamental.

Ejemplar de referencia: G. Giraldo $140 b$ (COL).

Nombres vernáculos: flor de sangre.

\section{Zephyranthes}

Hierbas perennes, $15-30 \mathrm{~cm}$ de altura. Bulbos de 1.5-5 cm de diámetro, solitarios o agrupados, túnica negra a marrón. Hojas 2-6, arrosetadas, espiraladas, sésiles, glabras, liguladas, ausentes durante la floración; lámina foliar estrechamente linear, $10-35 \mathrm{~cm} \times 0.2-1 \mathrm{~cm}$. Flor solitaria, terminal; escapo fistuloso, 9-30 cm de largo, con 1 o varias brácteas membranáceas, connadas y tubulares en la base, separadas distalmente por debajo del pedicelo. Flor actinomorfa, fragante, pedunculada, ocasionalmente sésil, erecta; perigonio infundibuliforme, tépalos 6, connados, desde la base, $3-5 \mathrm{~cm} \times 1.2$ $2 \mathrm{~cm}$, blancos, amarillos, lila, rojos, rosados; pedicelo 1-3.2 cm de largo. Estambres 6, libres; filamentos insertos, anteras largas, amarillas. Ovario ínfero, trilocular. Frutos cápsulas loculicidas, membranáceas. Semillas numerosas, aplanadas, con testa marrón a negra.

Cuatro especies están presentes en Colombia.

\subsection{Zephyranthes albiella Traub.}

Nativa, cultivada. 350-1000 m s. n. m.

Distribución: nativa de Colombia y Ecuador, descrita a partir de bulbos recolectados en Cundinamarca. Crece en bosques tropicales del valle del río Magdalena.

Departamentos: Bolívar y Cundinamarca.

Usos: ninguno conocido.

Ejemplar de referencia: $A$. Beuther 128 (COL).

Nombres vernáculos: lirio.

\subsection{Zephyranthes carinata Herb.}

Naturalizada, adventicia. $1700-2600$ m s. n. m.

Distribución: descrita originalmente de México, distribuida ampliamente en América y cultivada en otros con- tinentes. En Colombia se encuentra en áreas de bosque premontano y montano bajo de las tres cordilleras.

Departamentos: Antioquia, Boyacá, Cundinamarca, Quindío, Santander y Valle del Cauca.

Usos: ninguno conocido.

Ejemplar de referencia: F. Alzate 4263 (HUA).

Nombres vernáculos: cebolleta, lirio, jacinto.

18.3. Zephyranthes puertoricensis Traub.

Nativa. 0-800 m s. n. m.

Distribución: La distribución conocida para esta especie incluye las islas del Caribe, Centroamérica y en Suramérica se encuentra en Colombia, Venezuela y Surinam. Poco frecuente en Colombia, registrada para áreas cálidas de bosque tropical, de la parte alta y baja del valle del río Magdalena.

Departamentos: Bolívar y Tolima.

Usos: ninguno conocido.

Ejemplar de referencia: E. Pérez-Arbeláez 3102 (COL).

Nombres vernáculos: ninguno conocido.

18.4. Zephyranthes rosea Lindl.

Nativa, cultivada. 0-1500 m s. n. m.

Distribución: Crece de forma natural en las islas del Caribe y algunas localidades de Centroamérica. En Colombia se encuentra en el Caribe insular y continental y es cultivada en el interior del país.

Departamentos: Bolívar, Cundinamarca, San Andrés y Providencia.

Usos: ornamental.

Ejemplar de referencia: $A$. Beuther 97 (COL).

Nombres vernáculos: cebolleta. 

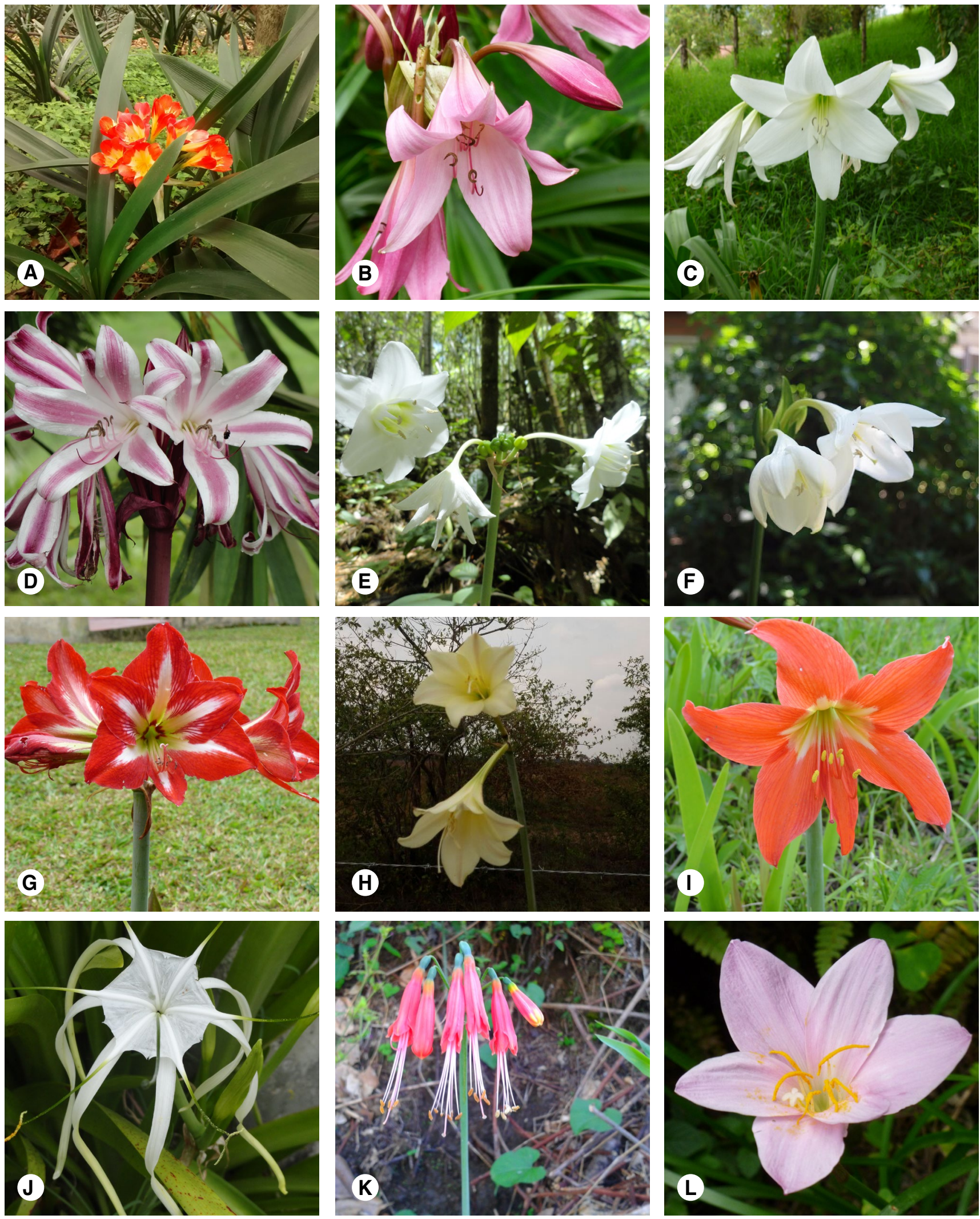

Figura 1. Algunas de las especies de Amaryllidaceae presentes en Colombia. A. Clivia miniata, B. Crinum bulbispermum, C. C. jagus, D. C. zeylanicum. E. Eucharis bonplandii, F. E. caucana, G. Hippeastrum barbatum, H. H. elegans, I. H. puniceum, J. Hymenocallis caribaea, K. Phaedranassa lehmannii, L. Zephyranthes carinata. Fotografías: Natalie Cortés y Fernando Alzate. 


\section{Discusión}

La diversidad de especies de la familia Amaryllidaceae en Colombia es muy baja si se compara con países como Brasil, donde se tiene reportada la ocurrencia de 142 especies y 19 géneros (Flora do Brasil 2020; http:/ / floradobrasil. jbrj.gov.br/reflora/floradobrasil/FB43). Sin embargo, la diversidad de este taxón es muy similar o mayor a la de áreas como Mesoamérica (Trópicos; http:/ / www.tropicos.org/Name/42000442? projectid=3\&langid=66), en la cual se tienen reportes de 8 géneros y 32 especies, o Ecuador con 11 géneros y 33 especies (Tropicos; http:/ / www. tropicos.org/Name/42000442?projectid=2).

La cordillera Occidental alberga la mayor diversidad de especies nativas de Amaryllidaceae en Colombia, en la cual se encuentran 11 géneros y 15 especies; en esta cordillera la mayor diversidad se encuentra en los departamentos de Cauca y Valle del Cauca, lo cual puede ser parcialmente debido a la intensa exploración botánica de estas áreas por parte del taxónomo P. Silverstone-Sopkin, especialista en la familia. En el Chocó biogeográfico y la cordillera Oriental se encuentran tres géneros y seis especies, en cada una de estas regiones. Es evidente la preferencia por ambientes húmedos y muy húmedos, en la mayoría de las especies de la familia que se encuentran en Colombia, mientras que en zonas como el sur de África, donde existe una considerable diversidad de Amaryllidaceae, la mayoría de las especies se encuentran adaptadas a ambientes secos y son deciduas (Strydom, 2005).

Eucharis es el género más diverso de la familia en Colombia, con ocho especies nativas, de las 17 conocidas para este grupo de plantas. Este taxón, restringido al Neotrópico, es más diverso en tierras bajas de la Amazonía y valles interandinos (Meerow, 1989) y su diversidad disminuye al aumentar en latitud. Con esta revisión se pudo determinar la alta diversidad de especies que alberga Colombia para este género, superado solo por la diversidad encontrada en Perú (Tropicos; http:/ / www.tropicos.org/Name/40006548? projectid=5).

Celis (2019) en el catálogo de plantas de Colombia, incluye a Eucharis castelnaeana (Baill.) J.F.Macbr. y presen- ta como espécimen representativo la colección Watson 1868 depositada en el herbario FLAS. Sin embargo, esta colección es más afín a E. ulei, considerando sus caracteres foliares, florales y su distribución geográfica, razón por la cual no fue incluida en esta sinopsis.

Nueve especies de la familia pertenecientes a cinco géneros son endémicas de Colombia. Las especies nativas en Colombia son en su mayoría muy raras o escasas, lo cual puede incidir en su vulnerabilidad a futuro. Varias de estas especies endémicas presentan serios riesgos para su supervivencia, como es el caso de Plagiolirion horsmannii, Eucharis caucana y E. lehmannii, para las cuales solo se conocen tres o cuatro localidades, con pocos individuos en cada población. La persistencia de estas especies es muy incierta, ya que, además de contar con poblaciones escasas, las áreas de presencia presentan alta presión de transformación de coberturas, debido a la intervención humana (Silverstone-Sopkin, 2012).

Cerca del $50 \%$ de especies de la familia (25) están representadas en Colombia por taxones cultivados con fines hortícolas, que en algunos casos se encuentran naturalizadas, como es el caso de Nothoscordum gracile y Zephyranthes carinata.

Stenomesson aurantiacum (Kunth) Herb. fue propuesta por Celis (2016) como "especie esperada" para Colombia, basado en su distribución conocida para el norte de Ecuador. Sin embargo, no se tiene ninguna colección de Colombia, por lo que su inclusión resultaría especulativa. Asi mismo, la especie Clinanthus incarnatus (Kunth) Meerow, endémica de Perú y Ecuador, se encontró cultivada en el Jardín Botánico de Bogotá, pero no se incluye en el inventario, ya que no se tiene evidencia de que se encuentre cultivada fuera de ese jardín botánico.

Zephyranthes susatana Fern. Alonso \& Groenend. solo es conocida de la colección tipo realizada en Suesca (Cundinamarca). Esta especie fue considerada por Celis (2016) como una población asilvestrada de Habranthus robustus. Sin embargo, varios caracteres del espécimen tipo, tales como como la posición erecta de la flor y las 
brácteas membranáceas, permiten identificarla como una especie de Zephyranthes, y posiblemente correspondiente a $Z$. carinata. Por esta razón no se incluyó esta especie en la sinopsis.

En general la familia carece de un tratamiento taxonómico y un análisis filogenético amplio y reciente que permita resolver los considerables problemas taxonómicos de relaciones y nomenclaturales, lo cual es más evidente en algunos géneros como Phaedranassa y Eucharis, que requieren una urgente revisión taxonómica. En cuanto a problemas nomenclaturales, buena parte de las especies de la familia no tienen definidas las colecciones tipo, debido parcialmente a la antigüedad de las descripciones, la escasez de información sobre las colecciones, el origen de estas y la no designación de tipos en los protólogos.

\section{Agradecimientos}

Este estudio fue desarrollado gracias al financiamiento de la convocatoria 727 de Doctorados Nacionales de Colciencias y al apoyo de CODI de la Universidad de Antioquia. Los autores expresan su agradecimiento a los curadores de los herbarios consultados, así como a Herber Sarrazola y Alex Espinosa (HUA), y muy especialmente al profesor Philip Silverstone-Sopkin (Q.E.P.D.) por sus valiosos aportes para mejorar este trabajo.

\section{Referencias}

APG I. (1998). An ordinal classification for the families of flowering plants. Annals of the Missouri Botanical Garden, 85(4), 531-553.

APG IV. (2016). An update of the Angiosperm Phylogeny Group classification for the orders and families of flowering plants: APG IV. Botanical Journal of the Linnean Society, 181, 1-20.

Beentje, H. (2016). The Kew plant glossary: an illustrated dictionary of plant terms. Kew: Royal Botanic Gardens.
Bernal, R., Galeano G., Rodríguez, A., Sarmiento, H. \& Gutiérrez, M. (2017). Nombres Comunes de las Plantas de Colombia. Recuperado de http:/ / www. biovirtual.unal.edu.co/nombrescomunes/

Bernal, R., Gradstein, S. \& Celis, M. (Eds.). (2015). Catálogo de plantas y líquenes de Colombia. Bogotá D. C.: Instituto de Ciencias Naturales, Universidad Nacional de Colombia.

Chase, M., Reveal, J. \& Fay, M. (2009). A subfamilial classification for the expanded asparagalean families Amaryllidaceae, Asparagaceae and Xanthorrhoeaceae. Botanical Journal of the Linnean Society, 161, 132-136.

Font Quer, P. (2001). Diccionario de botánica. Barcelona: Ediciones Península.

Idárraga A., Ortiz, R., Callejas, R. \& Merello, M. (2011). Flora de Antioquia: catálogo de las plantas vasculares. vol. II. Listado de las plantas vasculares del departamento de Antioquia. Programa Expedición Antioquia-2103. Series Biodiversidad y Recursos Naturales. Bogotá D. C.: Universidad de Antioquia, Missouri Botanical Garden \& Oficina de planeación departamental de la gobernación de Antioquia, D'Vinni. Pp. 1-939.

Meerow, A. (1989). Systematics of the Amazon Lilies, Eucharis and Caliphruria (Amaryllidaceae). Annals of the Missouri Botanical Garden, 76(1), 136-220.

Meerow, A. \& Snijman, A. (1998). Amaryllidaceae. En Kubitzki, K. (Ed.). The Families and Genera of Vascular Plants III. Pp. 83-110. Berlin: Springer-Verlag.

Silverstone-Sopkin, P. (2012). Los muertos vivientes: La historia natural de cuatro lirios amazónicos del suroccidente de Colombia (Eucharis y Plagiolirion, Amaryllidaceae). Cali: Programa Editorial Universidad del Valle. 98 pp.

Strydom, A. (2005). Phylogenetic relationships in the family Amaryllidaceae. (Trabajo de grado). Bloemfontein: University of the Free State, Faculty of Natural and Agricultural Sciences, Department of Plant Sciences, Genetics. 559 pp.

Thiers, B. (2018). Index Herbariorum: A global directory of public herbaria and associated staff. New York Botanical Garden's Virtual Herbarium. Recuperado de http://sweetgum.nybg.org/science/ih/ 


\section{Fernando Alzate}

(autor de correspondencia)

Universidad de Antioquia,

Facultad de Ciencias Exactas y Naturales

Medellín, Colombia

alveiro.alzate@udea.edu.co

https://orcid.org/0000-0002-4916-8897

\section{Manuela Lesmes}

Universidad de Antioquia,

Facultad de Ciencias Exactas y Naturales

Medellín, Colombia

manuela.lesmes@udea.edu.co

https://orcid.org/0000-0003-1503-3422

\section{Natalie Cortés}

Universidad de Antioquia

Medellín, Colombia

charlotte08@gmail.com

https://orcid.org/0000-0001-8895-6282

\section{Santiago Varela}

Universidad de Antioquia

Medellín, Colombia

aaronsantiago.varela@udea.edu.co

\section{Edison Osorio}

Universidad de Antioquia

Medellín, Colombia

edison.osorio@udea.edu.co

http://orcid.org/0000-0002-9821-3420

\section{Sinopsis de la familia Amaryllidaceae en Colombia}

Citación del artículo: Alzate, F., Lesmes, M., Cortés, N., Varela, S. \& Osorio, E. (2019). Sinopsis de la familia Amaryllidaceae en Colombia. Biota Colombiana, 20(1), 2-20. DOI: 10.21068/c2019.v20n01a01.

Recibido: 27 de marzo de 2018

Aceptado: 6 de mayo de 2019

20 | Biota Colombiana 20 (1) - 2019 